\title{
A User and Stakeholder-Driven Approach for Cross-Border, Seamless and Personalised MaaS Provision
}

\author{
Maria Gkemou $^{1(\varangle)}$, Maria Anna Devetzoglou ${ }^{1}$, Katerina Touliou ${ }^{2}$, \\ Athanasios Salamanis ${ }^{3}$, and Evangelos Bekiaris ${ }^{2}$ \\ 1 Centre for Research and Technology Hellas, Hellenic Institute of Transport, Egialias Str. 52, \\ Marousi, 15125 Athens, Greece \\ \{mgemou, mdevetzoglou\}@certh.gr \\ 2 Centre for Research and Technology Hellas, Hellenic Institute of Transport, \\ 6th km Charilaou - Thermi Rd, 57001 Thermi, Thessaloniki, Greece \\ \{touliouk, abek\} acerth.gr \\ 3 Centre for Research and Technology Hellas, Information Technologies Institute, \\ 6th km Charilaou - Thermi Rd, 57001 Thermi, Thessaloniki, Greece \\ asaleiti.gr
}

\begin{abstract}
Mobility as a Service (MaaS) is the new transport paradigm where service, transport and technology providers collaborate to deliver a seamless MaaS experience to travellers through the MaaS aggregator/issuer who, frequently, tackles the full service provision; from pre-trip planning to redemption. This manuscript presents the exploration of the design, the implementation and primary usability evaluation of an all-inclusive one stop shop that facilitates the transition to seamless MaaS, elaborating on the supported functions as well as the user/stakeholder centric design of the solution. Hybrid trip planning, matchmaking and personalisation, mobility tokens, business rules and back-office synergies collaborate to lead to a single Mobility Token of combinational MaaS packages for travellers to purchase and use. This work has been conducted in the context of MyCorridor $\mathrm{H} 2020$ project, with one of its key results being the recommendations towards a cross-border, inclusive and standardised MaaS.
\end{abstract}

Keywords: User and stakeholder driven MaaS · Personalised cross-border MaaS · One-stop-shop

\section{Introduction}

Increasing population, changing transport habits, growing demand of citizens for better transport and for integrated services and the rise of disruptive technologies have led to the consideration of the rapidly growing concept of Mobility as a Service (MaaS) as a cornerstone for a personalised, flexible and on-demand future of mobility. MaaS aims to urge private and public transport operators to integrate different forms of transport into one single, on-demand mobility service, placing travellers at the centre of transport services and providing them with customised mobility solutions.

(C) Springer Nature Switzerland AG 2020

B. Müller and G. Meyer (Eds.): Towards User-Centric Transport in Europe 2, LNMOB, pp. 254-266, 2020.

https://doi.org/10.1007/978-3-030-38028-1_18 
Despite the increasing penetration of $\mathrm{MaaS}$ worldwide, there are still issues unsolved. Some indicative are those tackling with penetration into vulnerable to exclusion populations, interoperability issues in relation to cross-border, multiservice and multi-context operation, policy, political and competition barriers that are hindering the foundation of an ideal formula for MaaS turning to be the mainstream way out, market "silos" that are hesitant to connect to MaaS solutions, data protection rules that make more sophisticated approaches hard to apply, lack of optimum business models that would hinder the creation of monopolies and, last but no least, technical implementation that lacks behind and is crucial for sustainable solutions.

The MyCorridor project, starting from the recognition of the ecosystem that would holistically compose the future MaaS value chain and putting it in the centre of its iterative design and evaluation workplan, proceeded with the definition of specific use cases addressing the needs and priorities of travellers but also all key participating stakeholders, coming up, finally, with the design, implementation and evaluation of an one-stop-shop for MaaS to materialise them. The one-stop-shop developed intends to be all-inclusive - addressing in a personalised manner the vast majority of potential travellers' needs - to work cross-border providing seamless mobility across Europe and bring together operators from public and private transport, mainly road, but also traffic management operators and operators of supporting to mobility added value services (i.e. touristic, entertainment, etc.). Travellers will interact with the one-stop-shop through mobile applications in Android and iOS, whereas service providers will be able to register their service in a semi-automatic manner through a web based service registration tool. All the above placed in the context of accommodating business models, data privacy policies and through payment and back-office services allowing a full MaaS service provision and its evaluation from mobility planning to single access mobility tokens towards redemption.

The paper starts with the presentation of the end-user and stakeholder-driven methodological approach applied for MaaS development and evaluation (Sect. 2) and the authors' perspective about the MaaS ecosystem (Sect. 3). In turn, the travellers' and stakeholders' needs and priorities are summarised in Sect. 4, whereas the identification of the key barriers, enablers and external megatrends impacting MaaS penetration follows in Sect. 5. Section 6 presents the Use Cases of an one-stop-shop developed for $\mathrm{MaaS}$, the technical approach supporting them and the mobility and other services feeding them. Section 7 provides recommendations towards a cross-border, inclusive and standardised MaaS upon the authors' experience gained and the manuscript concludes in Sect. 8 with some first usability evaluation results and the further steps anticipated.

\section{Methodological Approach Towards End-User and Stakeholder-Driven Use Cases for MaaS}

A stakeholder-driven iterative approach was applied for the final extraction of the Use Cases that would orient the one-stop-shop implementation as reflected in Fig. 1. Following the definition of the MaaS ecosystem, the first phase consisted of the investigation of relevant strategic priorities and policies that define the limits and priorities of operation, 
the analysis of the MaaS landscape to comprehend current and emerging MaaS solutions and recognise best practices and the investigation of traveller and other stakeholders needs, views and priorities through literature surveys, on-line surveys, interviews, focus groups and workshops. In a second phase, the key success and failure factors for MaaS deployment were identified and the impact of external trends was explored. In turn, MaaS impacts were evaluated using impact assessment results of previous initiatives, developing at the same time the reference Key Performance Indicators (KPIs) for MaaS success. Finally, all collected findings were processed to lead to the determination of user and market driven requirements serving as a clearance checklist of the project Use Cases and application scenarios. The latest were the key tool guiding the design and implementation phase of the one-stop-shop. Apart from the system use cases, an initial pool of the clusters of services that would be required to feed the one-stop-shop under the vision of a cross-border seamless mobility provision was structured.

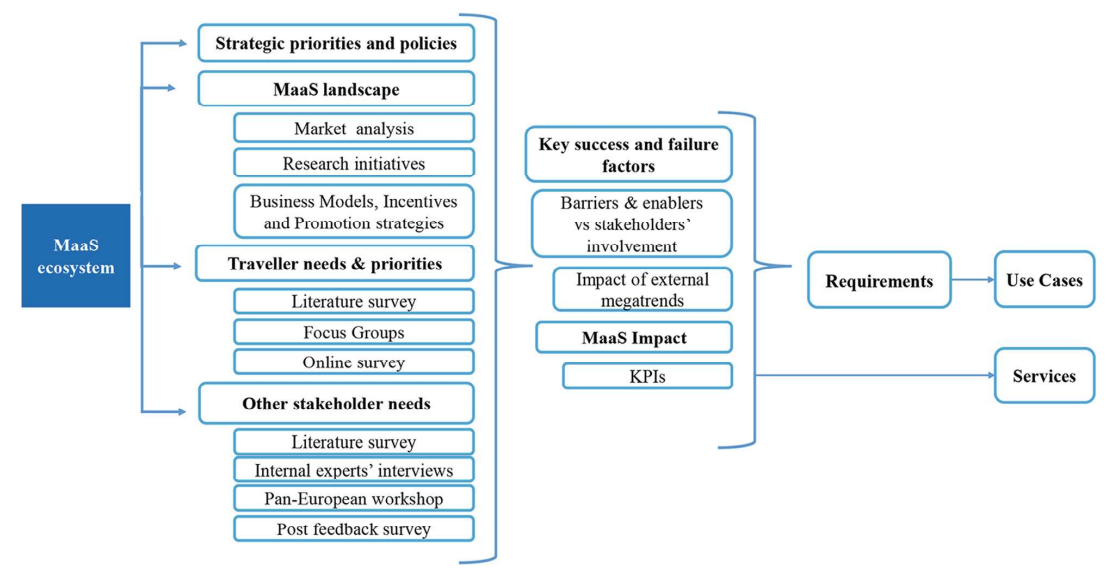

Fig. 1. MyCorridor methodology towards determining the Use Cases of the one-stop-shop

\section{The MaaS Ecosystem}

MyCorridor has further built on the classification proposed by the MAASiFie project [1], perceiving a MaaS ecosystem consisting of the following actors:

Government/Authorities (Ministries of transportation, transport agencies, road administration and transport safety authorities) with a legislating role, acting frequently as financial instruments, implementing transport policies, strategy and investments, responsible for the strategic transport planning and the management of the national transport infrastructure.

Cities/Regions (regional/local transport entities, tourist agencies, city planning departments), responsible for the strategic urban planning and urban infrastructure, managing Public Transport (PT) and, frequently, urban traffic management. 
Mobility/MaaS aggregators/issuers who may originate from the public, private or public-private sector, being a single or an alliance of aggregators/issuers, being responsible for the centric MaaS provision over a defined region.

Transportation service providers/operators, from public or private sector, operating traditional or new-coming road or multimodal mobility services or services associated or supporting mobility, using Intelligent Transport Services (ITS) or not, being single transport providers or service aggregators themselves, including Traffic Management operators.

Mobile service providers, including infomobility, telecom, ITS and Information and Communication Technology (ICT) providers, dynamic navigation service providers, payment and ticketing facilities and various trusted $3^{\text {rd }}$ parties.

Travellers, of all cohorts, being the MaaS consumers, encompassing pedestrians, cyclists, PT passengers, car drivers but also vulnerable to exclusion travellers (people with disabilities, elderly, with language barriers, etc.).

\section{Analysis of Travellers' and Stakeholders' Needs}

\subsection{Travellers' Needs}

To identify traveller needs, a literature survey was conducted complemented by focus groups and an on-line survey, collecting input by 186 participants from 18 countries in total. To better segment the results collected whenever possible, the following traveller profiles were pre-identified: daily commuter, tourist, businessman/businesswoman, spontaneous user, mobility-restricted user, medium IT literacy user and "bleisure" traveller (traveller who combines business with pleasure).

According to the literature survey results consolidating input from around 35 sources, the majority of travellers (78\%) use their smartphones while using PT whereas 70\% of Londoners were reported to regularly use apps to plan their journey [2]. Moreover, travellers prefer using one single access app to book their tickets. Although according to the consolidated results, privacy and personal data protection were the key user concerns, though 57\% of the former UK's Transport Catapult survey respondents would not mind sharing their personal data to get better transport services [3]. The focus groups were attended by 44 participants in Athens (10 participants), Brussels ( 7 participants), Prague (8 participants), Rome (5 participants), Salzburg (8 participants) and Thessaloniki (6 participants). Focus groups brought together users with different profiles - responding to the pre-defined profile clusters and raised specific topics, namely: human experience, conceptual acceptability, technology and legal concerns. Participants revealed that their mobility decisions depend on a number of factors, such as their location, service availability and reliability, weather conditions, convenience, reason and context for transport. Participants perceive MaaS as a helpful means mainly for when travelling abroad, rather than their everyday mobility. In addition, the culture of ownership is still quite strong with many participants being sceptical whether PT reliability and comfort level are adequate enough to urge them abandon their vehicles. 
An online survey via Sosci Survey (https://www.soscisurvey.de) was also promoted through the project's web site, social media and the Consortium beneficiaries' networks targeting travellers from different countries, age groups, gender and socio-economic backgrounds. The survey tackled mobility habits, needs, preferences and priorities and was completed by 142 participants in total. The majority of travellers belong in the 25-44 age group while participants came from Greece (22\%), UK (19\%), Italy (18\%), Austria (14\%), Netherlands (8\%), Germany (4\%), France (2\%), Belgium, Czech Republic, Finland, Hungary, Norway, Portugal, Russia, Slovakia, Slovenia, Spain and Sweden (each $1 \%$ ), offering a rather good distribution amongst European countries considering the habits, service, infrastructure and mentality around mobility. $80 \%$ consider themselves confident users of technology while $66 \%$ stated they already use web/mobile applications to support their mobility. Mobility habits wise, $70 \%$ stated they own a car, $12 \%$ own a motorcycle and an impressive $70 \%$ owns a bicycle, making it a highly popular means of transport. In terms of PT use, 55\% replied they rarely use it in contrast with $43 \%$ who often use it. $2 \%$ of the travellers declared they never use PT due to insufficient network, low level and unreliable services. $76 \%$ were familiar with mobility schemes such as car/ridesharing and carpooling, although only $34 \%$ have used any of them. Out of the users of such services, $31 \%$ use it on a monthly basis, while $15 \%$ have used it for cross-border journeys. The above results demonstrate the growing trend of travellers to turn to PT provided services available for them are reliable as well as opting to share, rather than own, private vehicles to avoid costs and congestion.

It has been also shown that for cross-border trips, reliability and cost are the more important criteria, whereas for local journeys, reliability and time are the ones that matter the most (Fig. 2). Regarding their preferred mode of transport, most participants prefer to use their private vehicle (46\%) on a local basis while when abroad they prefer to use PT (68\%). Moreover, $75 \%$ would be willing/very willing to change mobility habits provided travel services were made better overall. The most crucial aspects that should be addressed in traveller's interaction with the one-stop-shop through mobile are security of transactions (13\%), UI friendliness and intuitiveness (13\%) and reliability of the info provided (12\%). Participants also showed reluctance to share other people's vehicles, share their own means of transport and trust the information provided by a MaaS onestop-shop, doubting the reliability of the information. Key benefits of a MaaS enabling system, as stated by participants, include independence, flexibility, time efficiency, cost effective journeys and convenience while drawbacks include concerns about reliability of information, security issues for vehicle sharing services and MaaS not being operational in case of lack of network (Fig. 3).

\subsection{Stakeholders' Needs}

The needs and priorities of the MaaS ecosystem stakeholders were identified via a literature and market survey, a Pan-European workshop gathering a number of them and a post-workshop feedback survey. The literature and market survey conducted led to the decomposition of the key profile and roles per each. Governments/authorities and Cities/Regions, formulate and apply the respective transport policies, strategies and regulations addressing all operational aspects in transport service delivery (public transport, public bike schemes, travel information etc.) [4]. They also decide which 


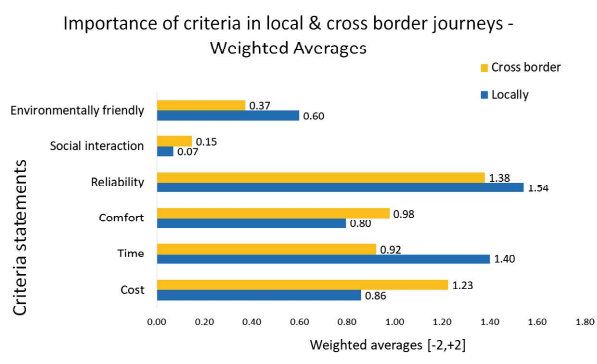

Fig. 2. Importance of criteria in local and cross-border journeys according to travellers' online survey.

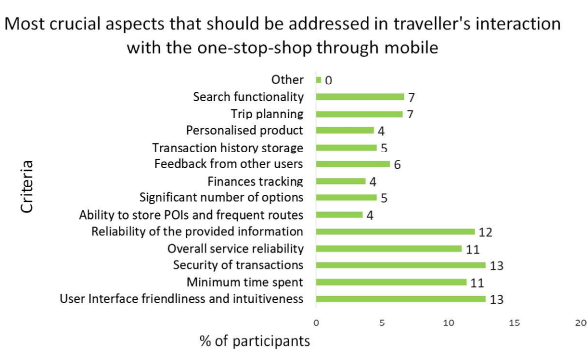

Fig. 3. Most crucial aspects that participants feel should be addressed in travellers' interaction with the one-stop-shop according to travellers' online survey.

business/operational models are viable anticipating their implications on the users, the business environment, the economy and the legislative frameworks [5]. It is of their outmost concern to have in place mobility operations being interoperable across different mobility platforms adhering to legislation and service agreements. Quite often, Public Private Partnerships (PPP) are promoted to provide added value services and increase user acceptance [6]. Mobility/MaaS aggregators/issuers are aggregating different mobility services into single-access platforms to support easy and tailor made traveller mobility [7], making use, for this purpose, Transport providers/operators data on schedules, fares and real-time feeds [8]. An Application Programming Interface (API) usually allows third parties to add their content [9] and encourage individual operators to easily join MaaS platforms [10], whereas transparent and viable Service Level Agreements (SLAs) between aggregators and operators/providers are required to ensure compliance with EU legislation. Mobile Service Providers provide all the enabling infrastructure and third party services to support MaaS [11], often are the ones providing building blocks that ensure interoperability, data security and sustainability of the technological MaaS platforms.

The above results obtained by the literature and market review were reinforced by a Pan-European workshop, that was held in the context of MyCorridor project in London in February $9^{\text {th }}, 2018$. A live Mentimeter interactive session encouraged feedback of 50 attendants addressing four main topics, namely MaaS \& public sector, MyCorridor Use Cases, Business Models and Towards a Euro-Mobility ticket. Overall, it became apparent the decisive role of Cities and authorities in MaaS deployment. Despite the fact that $52 \%$ of the attendants claimed that the main driver for MaaS success should be market-driven rather than policy-driven; still, $45 \%$ of them agreed that the main barrier relates to regulatory frameworks for the harmonisation of which, authorities undoubtedly play the key role (according to $32 \%$ of the attendants). Contracts/agreements and financial incentives/disincentives are also important in this (according to $23.5 \%$ and $24.5 \%$ respectively). Agreeing more or less with the travellers' survey outcomes (Fig. 2), time and cost were again considered as the most important mobility criteria for travellers (according to $69 \%$ and $75 \%$ of the participants respectively), followed by comfort (40\%) and environmental friendliness (29\%). The concept of the Euro Mobility ticket was also introduced in the workshop. That is a single access digital pass serving 
as a compendium of all individual mobility tokens, ready for use by the traveller, acting as a precursor of the integrated ticket. The majority of stakeholders (62\%) found this concept as a necessary solution, stressing the obvious regulatory barriers for its adoption. Finally, stakeholders stated the importance of transparent, viable and mutually beneficial SLAs with all providers to ensure secure data flow and sharing, service reliability and interoperability, according to policy guidelines and standards.

In addition to the live feedback collected, a post-workshop feedback online survey was completed by 20 of the participants with different professional backgrounds (transport engineers, ICT researchers, civil engineers) originated from the Czech Republic $(20 \%)$, Greece $(40 \%)$ and Ireland (20\%) with a $20 \%$ of them not disclosing country information. The post-workshop feedback online survey addressed MaaS enablers/barriers, Business Models and potential Use Cases. Participants prioritised scaling discounts, promotion campaigns and loyalty schemes to be the top three strategies in stimulating MaaS business success while the top three strategies for encouraging environmentally friendly mobility were scaling discounts, environmental benefits per model shift and tax reductions. Regarding the Use Cases of a MaaS platform, personalisation, matchmaking (mapping user's profile to mobility products suggestions) and back-offices synergies ranked first, whereas the availability of a significant number of options for the traveller was considered the most crucial element of a MaaS platform.

\section{Barriers, Enablers and External Megatrends}

One of the key outcomes of the post-workshop survey was the prioritisation of the enablers and barriers for the deployment of MaaS services that had been identified earlier, across six (6) broad categories, namely User-market related, Conceptual, Technological, Organisational, Business-related and Legal [12]. 21 barriers and 18 enablers had been identified in total. Each enabler/barrier impact priority was calculated as weighted average value of the aggregated questionnaires results of the post-feedback survey. It seems that those barriers related to the collaboration between stakeholders, the interoperability and compatibility, the security and data management, the new roles to be undertaken by the public administration and the new and still unclear business models ambiguous adoption overall, are the ones recognised to have the greatest impact in MaaS development and penetration. On the other hand, the enablers that are expected to have the greatest impact are namely the higher level of end user's involvement, the increased attention for sustainable transport, the need for flexible and personalised services, the increase of reliable traffic information and those related to organisational aspects, such as the MaaS alliance activity, the universal mobility offers, the progress of cooperative ITS data policy in Europe, more cooperation between stakeholders and the activity of the Smart Ticketing Alliance as well as the political pressure for change and the PPPs enabling value.

Moreover, four (4) external key megatrends were recognised to impact on MaaS deployment in the medium term as follows: urbanisation, sustainability (environmental challenges), demography and travel trends towards multimodality. Considering that $75 \%$ (and rising) of EU's population lives in urban agglomerations and 85\% of EU's GDP (Gross Domestic Product) is generated in urban areas, urbanisation is expected to pose 
a major threat to cities, such as increase of congestion, land use and pollution, impacting negatively on citizens' quality of life. Sustainability-wise, transportation is responsible for $20 \%$ of all $\mathrm{CO}_{2}$ emissions with around $75 \%$ being caused by road transport, indicating that MaaS is expected to play a major role in $\mathrm{CO}_{2}$ emissions reduction in transport (e.g. the shift from private modes to public and active modes supports the targets for declining emissions) [13]. Demographics changing will create further opportunities for MaaS solution for senior citizens and vulnerable road users while the global 15-34 age group are important customers of the future and, specifically in favour of MaaS, as they are tech savvy and connected $24 / 7$, demanding, impatient, civic and environmental friendly [14]. Finally, multimodality is another mega mobility trend, as young adults' tendency to use multiple modes of transport has been on the rise in recent years [15].

\section{Use Cases, Services and Technical Approach for a MaaS One-Stop-Shop}

\subsection{Use Cases for MaaS One-Stop-Shop}

As delineated in Fig. 1, the key outcome of all the user and stakeholder centric exploration activities was the establishment of the Use Cases for MaaS one-stop-shop (that would be developed in the context of MyCorridor project), aiming to reflect its main functions [12]. Travellers' interaction with the one-stop-shop will be made through the mobile application developed for this purpose, named "MyOSS", that is available in Android and $\mathrm{iOS}$, while service providers will interact with the one-stop-shop through a webbased Service Registration Tool. The UCs of the one-stop-shop for the travellers are described below in short:

T1. User Login/Register/Authentication. This UC reflects new traveller registration and sign up, login, failed registration, authentication and login of unregistered user. T2. Static \& semi-dynamic profiling. This UC covers the creation of a profile by a new, unregistered or already registered user. The level up to which users denote preferences defines the level of personalisation of the MaaS service delivered to them. Travellers can set-up and edit their profile denoting their information, accessibility preferences, cost preferences, comfort, preferred transport modes, preferred payment method, hobbies, interests, etc. Static information (information that does not change in time, i.e. name, gender, language) can be changed only by the user through the preferences whereas semi-dynamic information (information that changes in real time, i.e. mode preferences, traveller profile) can be updated while the user interacts with the application (e.g., in the context of configuration, purchase and redemption of Mobility Products offered for selection and use by the one-stop-shop). T3. Personalised MaaS package configuration, purchase \& redemption. T3 addresses the configuration, purchase \& redemption of personalised MaaS packages (consisting of one or more mobility products) coupled with trip planning or configure upon a sophisticated multicriteria search (without encompassing trip planning). The end-output of the interaction with the onestop-shop is a single access consolidated Mobility Token that includes all the eligibility criteria of the mobility products tickets and is ready for redemption/validation at the 
operators/providers side. Upon the redemption of Mobility Tokens and the loyalty policy in place, travellers are able to collect points or credit. T4. Personalised Info support (added value services - athletic, touristic, cultural, health push personalised notifications) and synthetic services. This UC is complementary to UC T3 and is optional for the traveller, as they may not wish to receive any push notification for added value services. Synthetic services, in specific, refer to new mobility products that are synthesised by the MaaS aggregator/issuer ad-hoc upon the mobility services availability and feasibility to combine and utilising popularity data from past use for specific traveller clusters. In a commercial operation, the business models that would be applied could be an additional driver for the type of synthetic services promoted and proposed. T5. Modification/Cancellation. This UC corresponds to modifying and cancelling selected mobility products and functions. T6. Traveller Feedback. This UC tackles the viewing of information that comes from other travellers, the sharing and provision of feedback through social media to other travellers and the provision of feedback on the MaaS experience as a whole. Furthermore, travellers can also view their favourite trips, trip and mobility products' history and review history. T7. Transactions and Loyalty scheme (encompassing incentivisation \& rewarding). UC T7 addresses the transactions that take place through MyCorridor leading to the loyalty points collected by travellers. Travellers can view their wallet, transaction history, rewards and the points available to redeem.

Service providers' interaction, on the other hand, is featured through the following UCs: S1. Service provider log-in. This UC addresses the service provider log-in, covering the login of an already registered service provider and the sign up and log in of a new/unregistered service provider. The service provider can login to the Service Registration Tool through the registration tool webpage to register their services. S2. Service registration. This UC reflects the registration of the service from the service provider to the registration tool. S3. Service provider business rules editing. This UC refers to any editing actions that the service provider may wish to perform on their service. The matchmaking module can use those business rules in order to offer the best personalised options to the traveller.

The MaaS aggregator/issuer UC's are as follows: B1. Overall Business Rules editing. This UC refers to the overall business rules editing that is performed by the MaaS aggregator for the Mobility Products offerings to the travellers, which will take into account the individual service providers' business rules. B2. Added value synthetic. It addresses the synthesis of existing Mobility Products into new single Mobility Products as described above. B3. Clearance with the traveller and the service providers (evouchers). This UC addresses the travellers' approval to proceed with payment and the creation, issuing and cancellation of e-vouchers. B4. Mobility Token Issue and redemption (use/validation). Upon completion of B3, procurement and back-office clearance is being held between the aggregator and the engaged service providers leading finally to the issue of the Mobility Token(s) for the traveller to validate. B5. Interactive Traffic Management Plan. This UC refers to the interaction and cooperation of different actors involved in the interactive Traffic Management Plan deployment through the implementation of the Traffic Management 2.0 (TM2.0) Innovation platform's framework (TM2.0, launched by ERTICO) on MaaS. 


\subsection{Services for the MaaS One-Stop-Shop}

The services integrated in the one-stop-shop built are clustered in four groups. The first group consists of mobility services and includes all services that result in any type of purchasable ticketing products. Vehicle related services (parking, rental, vehicle sharing/pooling), PT services of all modes (urban, interurban, para transit) and peripheral mobility services (park\&ride, parking) are included. Traffic Management services encompass services available in each pilot site, related to real time traffic conditions, traffic forecast, incident management, access control and tolling, urban congestion pricing, zone access control and C-ITS enabled traffic management. The key innovation here is the application of the TM2.0 paradigm in MaaS. Infomobility services cover all services related to the information and real-time support of the user in pre-trip phase (trip planning), on-trip phase (notifications on itineraries schedules and their real-time updates, near parking availability) and after-trip phase, if applicable. Added value services refer to all services that give an additional value to travellers' mobility and improve travel experience, regardless of their association to mobility. They may include touristic, cultural and entertainment services (i.e. provision of events touristic/cultural/entertainment information) and new combined services that result from the ad hoc combination of available services in the one-stop-shop on behalf of the MaaS aggregator. All the services belonging to the clusters aforementioned are aggregated into the one-stop-shop and are offered to the travellers as MaaS packages across the different types of products anticipated and, as evident from the above description, their type ranges from purchasable mobility products, to infomobility or added value services.

\subsection{Technical Approach}

The back-end of the MaaS platform that was built in order to satisfy the use cases designed for it, follows a multi-tier architecture. This architectural style offers many advantages to complex platforms, such as the separation of concerts principle, according to which, components within each layer deal only with the logic of their layer. This feature increases flexibility, maintainability and scalability. Within this multi-tier system architecture, a series of system components have been designed and implemented, the key ones of which are namely: the Mobile Application which is the front-end module of the one-stop-shop serving for travellers' interaction with it, available in iOS and Android operating systems; the Web Applications through which the service providers and the MaaS aggregators get access to the one-stop-shop (the Service Registration Tool (SRT) and the MaaS Aggregator Dashboard respectively); the Hybrid multimodal trip-planner supporting all potential trip requests; the Matchmaking Module being responsible for matching the traveller's requests with the MaaS service offerings available in the one-stop-shop; the Multi-criteria Search Module which is responsible for retrieving services according to different user search criteria (e.g. transportation module, type of mobility product, etc.); the MaaS Product Synthesis Module responsible for supporting the generation of new services from the MaaS aggregator as the outcome of synthesis/combination of two or more different services; the Traveller Feedback Module responsible for integrating the travellers' feedback, regarding either the individual mobility services or the combined MaaS packages being used; the Big Data Management Module which provides the data 
analytics services that produce useful insights regarding the usage of the MaaS services and products; the Business Rules Implementer Module which provides the necessary functions to the service providers and the MaaS aggregator for viewing, modifying and validating the business rules of the individual services and the overall one-stop-shop; the Payment Module which is responsible for the payment of the different service providers through the dedicated payment facility services as well as the integration with the backoffice systems of the underlying service providers, in order for the traveller to be able to select, pay and receive the desired MaaS service; the Travellers' Data Repository that holds all data entities related to the traveller; the Services' Data Repository that holds all data entities related to the services and the service providers, and, finally, the MaaS API which is a stable, robust, efficient and secure RESTful API that is responsible for the communication and interaction between all the one-stop-shop components as well as for the communication between the one-stop-shop and the external modules (e.g. Traffic Management Services Aggregator).

\section{Recommendations Towards a Cross-Border, Inclusive and Standardised MaaS}

In the context of the operation of a MaaS ecosystem, interoperability issues may arise concerning either the seamless service provision itself and/or the management of data and processes. The former type of interoperability is referred to as service interoperability, while the latter as data interoperability. The experience gained through the research, development and evaluation phases presented in this manuscript allows the issue of some primary recommendations with respect to those matters.

The concept of service interoperability is related to the seamless provision of mobility solutions from a MaaS platform, in the case that the traveller requests a trip that crosses the border of a city, region or a country. In such cases, specific challenges may arise which can be considered as barriers in the wider acceptance of the MaaS mobility paradigm. The key technical approach to tackle this as it has been implemented and primarily evaluated in this research work, lies in splitting the trips into "legs", with each of them being operated on an individual basis. This approach reduces the probability that the start and end points of a defined part of a trip (the so-called "leg") being in different sides of a border, thus reducing the probability that the cross-border interoperability issues arise. However, this solution comes with the technical/computational cost that the matchmaking process should be conducted for each leg of each of the trips generated with a trip request from a traveller. In the MaaS one-stop-shop discussed herein, this technical limitation is handled, in the implementation level, by the use of a high performance programming language (i.e. $\mathrm{C}++$ ) which inherently offers appropriate tools (e.g. OpenMP, POSIX Threads, etc.) for easily parallelising the matchmaking process taking place for the legs of a trip. This process can be considered as very close to embarrassingly parallel, as the consecutive legs of a trip share only one common point (i.e. the end point of a leg is the start point of the immediately next leg).

The data interoperability issues of a MaaS platform refer to the several different data formats/templates/structures that exist in the transport industry for the representation of the same data entities that usually appear in the operation of a MaaS platform (e.g. trips, 
services, payment transactions, etc.). This heterogeneity in the representation of the data creates difficulties mainly in the service registration and the services interconnection processes within a MaaS platform, both in technical and in business level. In the current research work, the data interoperability issues are handled in two complementary ways, which are also proposed by the authors for the design and implementation of a complete data specification for MaaS, as follows:

- By designing and implementing MaaS specific technology (e.g. Matchmaking Module, Payment Module, etc.).

- By defining a complete set of most of the data models involved in the operations of a MaaS platform, and implementing the realizations of these data models using two different approaches, namely as JavaScript Object Notation (JSON) - like documents and as W3C Web Ontology Language (OWL) ontologies.

\section{First Usability Results and Next Steps}

The usability of the one-stop-shop mobile application has been already evaluated in controlled usability tests in laboratory setting through the completion of scenario-based tasks and "think-aloud" technique providing evidence about the effectiveness and value of the use cases, and, finally, the functionalities designed for it. Tests have been conducted in five (5) European countries (Austria, Czech Republic, Greece, Italy, and The Netherlands). The travellers participating in the Greek pilots consisted of eight (8) male $(44 \pm 7.3$ years old) and five (5) female (43 \pm 5.6 years old) with above average ICT literacy, all of them owning an Android mobile phone. Users were able to create an account, set their own travelling preferences and profile and configure their MaaS products. Across scenarios, perceived ease of use (64\%), usefulness $(66 \%)$ and acceptance $(68 \%)$ are above average (with similar results from the other Pilot sites) implying that the initial design of the mobile application is usable and acceptable by the potential users. Overall, task completion rates were $76 \%$, supporting the acceptable usability of the application through both performance and subjective measures.

Future steps include the optimisation of the one-stop-shop front and back-end, the completion of all functions required and the real-life evaluation of it in Pilots organised across the same sites. Real life pilots are expected to start in autumn 2019, targeting the participation of 15 service providers and 300 travellers in total. Travellers will download the MaaS application in their iOS or Android smart phones and will use it to plan, configure, book, pay and realise their trips, making use of real and different mobility and added value services integrated in the one-stop-shop that will be provided to them in personalised MaaS packages accompanied by single access Mobility Tokens ready for redemption. Pilots will be performed in local, regional and cross-border context in the boundaries and across the pilot sites aforementioned. The results that will be partially collected through web/mobile analytics and, partially, through the back-end of the onestop-shop, complemented at the end by local stakeholder focus groups, will allow one of the first in-depth impact assessment studies of medium scale in MaaS in addition to providing findings on the acceptance and performance of the specific MaaS solution developed in the context of this research. 
Acknowledgements. The work presented in this manuscript has been realised in the context of MyCorridor (Mobility as a Service in a multimodal European cross-border corridor) project which has received funding from the European Research Council (ERC) under the European Union's Horizon 2020 research and innovation programme (Grant Agreement $n^{\circ}$ 723384).

\section{References}

1. Konig, D., Eckhardt, J., Aapaoja, A., Sochor, J., Karlsson, M.: Deliverable 3: business and operator models for MaaS. MAASiFiE project funded by CEDR (2016)

2. Swiss Federal Railways. https://www.sbb.ch/en/home.html

3. Transport Systems Catapult: Mobility as a Service: Exploring the opportunity for mobility as a service in the UK (2016). https://ts.catapult.org.uk/wp-content/uploads/2016/07/Mobilityas-a-Service_Exploring-the-Opportunity-for-MaaS-in-the-UK-Web.pdf

4. Polis Traffic Efficiency \& Mobility Working Group: Mobility as a Service: Implications for urban and regional transport (2017)

5. Tourism and Transport Forum Australia, UITP: Mobility as a Service: the next transport disruption (2017)

6. UK Government Office for Science, MaaS Roundtable: Fore-sight Future of Mobility project (2017)

7. Gkemou, M., Kvaček, F.: Emerging MaaS schemes and multi modal platforms. Internal Report, MyCorridor project, G.A. 723384 (2017). http://mycorridor.eu/

8. Eckhardt, J., Aapaoja, A., Nykanen, L., Sochor, J., Karlsson, M., Konig, D.: Deliverable 2: European MaaS Roadmap 2025. MAASiFiE project funded by CEDR (2017). http:// publications.lib.chalmers.se/records/fulltext/249639/local_249639.pdf

9. Hietanen, S.: Mobility as a Service - The End of Car Ownership? (2017)

10. Li, Y., Voege, T.: Mobility as a Service (MaaS): challenges of implementation and policy required. J. Transp. Technol. 7, 95-106 (2017). https://doi.org/10.4236/jtts.2017.72007

11. Euronews article: Which European commuters spend the most time in traffic jams? (2018). http://www.euronews.com/2018/02/07/which-european-commuters-spend-the-mosttime-in-traffic-jams-

12. Gkemou, M., et al.: Deliverable D1.1: MyCorridor Use Cases, MyCorridor (Mobility as a Service in a multimodal European cross-border Corridor) project, G.A. 723384 (2018) http:// mycorridor.eu/

13. Silva, M., Ribeiro, N., Rodrigues, M.: Race 2050: trends in the demand for transportation systems. WIT Trans. Ecol. Environ. 191, 885-897 (2014)

14. Schroten, A., et al.: Deliverable D2.1: Assessment of the main barriers and KPIs for the implementation of ITS services, NEWBITS (New business model for ITS) project, G.A. 723974 (2017). http://newbits-project.eu/

15. Kuhnimhof, T., Buehler, R., Wirtz, M., Kalinowska, D.: Travel trends among young adults in Germany: increasing multimodality and declining car use for men (2012). https://www. sciencedirect.com/science/article/pii/S0966692312001317 


\section{Author Index}

A

Abraham, Michael, 240

Al Haddad, Christelle, 117

Antoniou, Constantinos, 117

B

Baldauf, Ulrich, 89

Bekiaris, Evangelos, 43, 254

C

Cekic, T., 16

Coosemans, Thierry, 151

Cuadrado Capitán, Faustino, 60

D

de la Cruz, M. Teresa, 28

Dellas, Nikolaos, 184

Devetzoglou, Maria Anna, 254

Döge, Norman, 240

E

Eryilmaz, Elif, 184

F

Fähndrich, Johannes, 184

Földesi, Erzsébet, 43

$\mathrm{Fu}$, Mengying, 117

G

Gautama, Sidharta, 102

Geerts, David, 102

Gely, Corentin, 135

Gillis, Dominique, 102

Gkemou, Maria, 254
Grandsart, Delphine, 102

Grignard, Arnaud, 89

H

Hadjidimitriou, Natalia Selini, 184

Hiemstra-van Mastrigt, S., 225

Hooftman, Nils, 151

J

Jammes, Thierry, 43

K

Keserü, Imre, 3

Kluge, Ulrike, 117

Knieling, J., 16

Knote, Thoralf, 169

Koukovini, Mariza, 184

Küster, Tobias, 184

$\mathbf{L}$

Lah, Oliver, 215

Landwehr, A., 16

Larson, Kent, 89

Le Mortellec, Antoine, 135

López Baeza, Jesús, 89

Lopez, Angel J., 102

Loukea, Matina, 43

M

Macharis, Cathy, 3

Marlier, Evelien, 102

Masuch, Nils, 184

Messagie, Maarten, 151

(C) Springer Nature Switzerland AG 2020

B. Müller and G. Meyer (Eds.): Towards User-Centric Transport in Europe 2, LNMOB, pp. 267-268, 2020. https://doi.org/10.1007/978-3-030-38028-1 
$\mathbf{N}$

Noennig, J., 16

Noennig, Jörg Rainer, 89

Noyman, Ariel, 89

$\mathbf{P}$

Panou, Maria, 43

Pappers, Jesse, 3

Peltomaa, Juha, 79

Pletat, Udo, 184

Ploetner, Kay Olaf, 117

$\mathbf{R}$

Randhahn, Annette, 169

Royo, Beatriz, 200

S

Salamanis, Athanasios, 254

Sanders, Kevin, 102

Saxe, Sebastian, 89
Schroeter, B., 16

Straubinger, Anna, 117

T

Tatum, K., 16

Theodoropoulos, Thodoris, 184

Touliou, Katerina, 254

Trentesaux, Damien, 135

Tuominen, Anu, 79

V

Val, Susana, 28

Valls, Lluís Alegre, 60

Van Kuijk, J. I., 225

Van Mierlo, Joeri, 151

Veeneman, W. W., 225

W

Weber, Vanessa, 89 